\title{
Optimization on response surface models for the optimal manufacturing conditions of dairy tofu
}

\author{
Ming-Ju Chen ${ }^{\text {a }}$, Kun-Nan Chen ${ }^{\text {b,* }}$, Chin-Wen Lin ${ }^{\text {a }}$ \\ a Department of Animal Science, National Taiwan University, Taiwan, ROC \\ ${ }^{\mathrm{b}}$ Department of Mechanical Engineering, Tung Nan Institute of Technology, No. 152, Section 3, Peishen Road, Shen Keng, Taipei 222, Taiwan, ROC
}

Received 2 January 2004; accepted 29 June 2004

\begin{abstract}
The purpose of this research was to create response surface models through regression on experimental data and to apply the Sequential Quadratic Programming (SQP) and Genetic Algorithms (GAs) on the models to obtain optimal processing conditions for dairy tofu. The two-stage effort of obtaining a surface model using response surface methodology (RSM), and optimizing this model using GAs or SQP techniques was demonstrated to be an effective approach. Both SQP and GAs techniques were able to determine the optimal conditions for manufacturing the probiotic dairy tofu. The conditions were $1 \%$ of glucono-delta-lactone (GDL), $0 \%$ of peptides level, 3\% of isomaltooligosaccharides (IMO) and 18\% of milk concentrations, and they were confirmed by verification experiments. Among the SQP and two GAs employed, the SQP, modified with the multi-start capability, is the most efficient one.
\end{abstract}

(C) 2004 Elsevier Ltd. All rights reserved.

\section{Introduction}

In recent years, there has been a worldwide increase in consumption of fermented milk, especially for those products with probiotics. Developing the new dairy products with probiotics could provide the varieties of selections for customers and might robust the market in dairy industry (Liu, Chen, \& Lin, 2002). The idea of probiotic dairy tofu was from glucono-delta-lactone (GDL) tofu and yogurt. This new product, containing probiotics and prebiotics, provides the texture of tofu, flavor of yogurt, as well as health benefits. In order to manufacture a good quality dairy tofu and understand the effect of different ingredients on the chemical, physical and microbial properties of this product, response surface models were developed to describe the combined

\footnotetext{
${ }^{*}$ Corresponding author. Tel.: +886 2 86625916x73; fax: +886 2 27324070

E-mail address: knchen@mail.tnit.edu.tw (K.-N. Chen).
}

effect of the factors and modern optimization techniques were applied to attain optimal conditions for the manufacturing process.

Response surface methodology (RSM) is a collection of statistical and mathematical techniques useful for developing, improving and optimizing processes. It usually contains three stages (Myers \& Montgomery, 1995): (1) design of experiments, (2) response surface modeling through regression, and (3) optimization. The main advantage of RSM is the reduced number of experimental trials needed to evaluate multiple parameters and their interactions (Lee, Ye, Landen, \& Eitenmiller, 2000; Porretta, Birzi, \& Vicini, 1995). The experimental data were utilized to build mathematical models using regression methods. Once an appropriate approximating model is obtained, this model can then be analyzed using various optimization techniques to determine the optimum conditions for the process. RSM was successfully used for applications in developing new edible gels (Chen \& Lin, 2002) and finding the optimum producing 
conditions of the dairy product Kou Woan Lao, which is produced by mixing milk and culture filtrates extracted from a fermented rice product and used to form milk curds and to enhance flavor (Weng, Liu, \& Lin, 2001).

Optimization theory consists of a body of numerical methods for finding and identifying the best candidate from a collection of alternatives without having to explicitly evaluate all possible alternatives (Reklaintis, Ravindran, \& Ragsdell, 1983). In the context of RSM, empirical (mathematical) models are built using regression techniques on the results of a selected set of experiments. A well fitted model represents, approximately, all possible experiments with their experimental factors within the preset bounds. Through the use of optimization techniques, the optimum of the model corresponding to the experiment with conditions that will presumably produce the best result can thus be found. The final step is to perform experimental verification based on the optimal, experimental conditions. Among the optimization techniques, the steepest ascent (or descent) is commonly used (see, for example, State-Ease, Inc., 2000), but the method is relatively inefficient and is a local optimization technique capable of finding only local optima. Genetic Algorithms (GAs), although even less efficient than the steepest ascent, are considered as global schemes. The Sequential Quadratic Programming (SQP) technique is very powerful and efficient, and with some modifications it can also perform global optimizations (Chen, 2003). Both SQP and GAs were studied in this research.

Genetic Algorithms are search procedures that imitate the natural evolution process and can be used for the computation of the global maximum or minimum of a function (Mitchell, 1996). Genetic Algorithms differ from other search techniques in that they search among a population of points and use probabilistic rather than deterministic transition rules. As a result, Genetic Algorithms search more globally (Wang, 1997). D'souza and Simpson (2003) utilized the so-called the non-dominated sorting Genetic Algorithm for product family design and optimization. Chen, Chen, and Lin (2003) optimized the viability of probiotics in a new fermented milk drink and concluded that the two-stage effort, obtaining a surface model and optimizing this model using the GAs, resulted in a useful method of finding an optimal set of process parameters.

A quadratic programming problem is an optimization problem involving a quadratic objective function and linear constraints. The Sequential Quadratic Programming method represents state-of-the-art in nonlinear programming methods (The Math Works Inc., 2000 ) and can be used to solve a series of quadratic programming problems approximating the original nonlinearprogramming problem. The SQP is a powerful tool but involves a complicated procedure. The theory behind the SQP can be found in most optimization textbooks, e.g. Arora (1989) and Haftka and Gürdal (1992), and will not be elaborated here.

The purpose of this research was to create response surface models through regression on experimental data and to apply the SQP and GAs on the models to obtain optimal processing conditions for dairy tofu.

\section{Experimental process and response surface modeling}

This chapter describes the pre-optimization stages, including design of experiments, experimental process and response surface modeling. Optimizations will be presented in the next chapter.

\subsection{Experimental process}

The dairy product under investigation is a new type of dairy tofu made from milk. The experimental process is described in the following subsections.

\subsubsection{Preparation of probiotic dairy tofu}

The samples were prepared using $12-18 \%$ (w/w) skim milk powder (Anchor Foods, New Zealand, protein $37.60 \%$, lactose $49.80 \%$ ). Reconstituted skim milk in deionized water was mixed with $0.3-1 \%$ of gluconodelta-lactone (GDL) and the prebiotics (peptides from casein, pancreatic digested, $0.0-1.0 \%$, Cheng-Fung Co., Taiwan; isomaltooligosaccharides, IMO, 0.0-3.0\%, Cheng-Fung Co., Taiwan). Then, the mixed samples (each of $200 \mathrm{~mL}$ ) were inoculated with $1 \%$ each of Lactobacillus acidophilus, Lactobacillus casei, Bifidobacteria bifidum and Bifidobacteria longum in $250 \mathrm{~mL}$ beakers covered with aluminum foil, and fermented for $12 \mathrm{~h}$ at $37^{\circ} \mathrm{C}$. The fermentation time and temperature were suggested by the preliminary tests, which revealed that dairy tofu with 12 -h fermentation at $37^{\circ} \mathrm{C}$ yielded better hardness and had higher viability of probiotics. The headspace volume of the fermentation was around $20 \%$ of the total volume.

\subsubsection{Cultures and medium performance}

Pure lyophilized cultures of $B$. longum (CCRC 14605), L. casei subsp. rhamnosus (CCRC 12321), B. bifidum (CCRC 11844) L. acidophilus (CCRC 14079) were purchased from the Culture Collection and Research Center, Hsinchu, Taiwan, ROC Lactobacilli MRS (deMan, Rogosa and Sharp) and Lithium propionate MRS agar (LP-MRS) were used as the selective media for Lactobacillus spp. and Bifidobacteria spp., respectively (Lapierre, Undeland, \& Cox, 1992).

\subsubsection{Determination of probiotic growth rate}

For the determination of the viabilities of the probiotics, the populations of Lactobacillus spp. and 
bifidobacteria spp. were measured as growth rates. The suitability of the media was tested by plating decimal dilutions of the probiotic cultures. Thus, a 1-g sample of each pure lyophilized culture was decimally diluted into sterile peptone water $(0.1 \%)$ and then $0.1-\mathrm{mL}$ aliquot dilutions were plated onto the different media, in triplicate. Plates of MRS agar were incubated aerobically for $72 \mathrm{~h}$ at $37^{\circ} \mathrm{C}$ to inhibit bifidobacteria. Plates of LP-MRS agar were incubated anaerobically $(72 \mathrm{~h}$ at $37^{\circ} \mathrm{C}$, GasPak System-Oxoid, Basingstoke, Hampshire, England). The population in colony-forming units (CFU) and the characteristics of the colonies were recorded for each medium.

The specific growth rate (GR) corresponding to each culture was calculated using the following equation:

$\mathrm{GR}=\frac{\left[\log \left(\mathrm{CFU}_{1}\right)-\log \left(\mathrm{CFU}_{2}\right)\right]}{t_{2}-t_{1}}$

where $\mathrm{CFU}_{1}$ and $\mathrm{CFU}_{2}$ are the CFU at time $t_{1}$ (fermentation for $0 \mathrm{~h}$ ) and $t_{2}$ (fermentation for $12 \mathrm{~h}$ ).

\subsubsection{Determination of hardness}

The hardness of samples was determined by testing 5 replicate samples on a TA-XT2i/5 Texture Analyser (Stable Micro Systems, USA) fitted with a $5 \mathrm{~kg}$ load cell. The gels were formed in glass containers $(50 \mathrm{~mm}$ diameter, $65 \mathrm{~mm}$ height) with $80 \mathrm{~mL}$ of mixed samples and tested using a cylinder probe with a flat-ended head of $20 \mathrm{~mm}$ in diameter at a fixed rate of $10 \mathrm{~mm} / \mathrm{s}$. The probe traveled $80 \%$ depth into the samples. Gel hardness was expressed as the force $(\mathrm{g})$ at the maximum peak of the force-time curve.

\subsection{Response surface modeling}

Before any experiment taking place, design of experiments were first performed. The Box and Behnken design (BBD) (Box \& Behnkin, 1960) is a three-level design based on the construction of a balanced incomplete block design. The BBD is an efficient option for fitting response surfaces using three evenly spaced levels (Myers \& Montgomery, 1995). A four-variable BBD with five replicates at the center point was selected to build response surface models. The coded and uncoded variables and their respective levels are shown in Table 1.

To carry out response surface modeling, the regression method was performed on experimental results to build mathematical models. The models were then formulated as an objective function in an optimization problem that was consequently optimized using optimization techniques to obtain the maximum viability of the probiotics and the maximum hardness of the product. The RSM procedure of the Design-Expert ${ }^{\circledR}$ software package (State-Ease, Inc., 2000) was used to
Table 1

Process variables and their levels in four variables-three levels of response surface design

\begin{tabular}{lccc}
\hline Independent variable & Symbol & Level \\
\cline { 3 - 4 } & & Coded & Uncoded \\
\hline GDL concentration (\%) & $X_{1}$ & -1 & 0.30 \\
& & 0 & 0.65 \\
Peptides concentration (\%) & $X_{2}$ & -1 & 1.00 \\
& & 0 & 0.00 \\
IMO concentration (\%) & $X_{3}$ & -1 & 0.75 \\
& & 0 & 1.50 \\
Skim milk concentration (\%) & $X_{4}$ & -1 & 0.00 \\
& & 0 & 1.50 \\
& & +1 & 12.00 \\
& & & \\
& & &
\end{tabular}

fit the experimental data to polynomial equations of order one through three to obtain coefficients. The following linear relationship achieved this.

$Y_{i}=f_{i}\left(X_{1}, X_{2}, X_{3}, X_{4}\right)+\epsilon_{i} \quad i=1,2,3$

where $Y_{1}, Y_{2}, Y_{3}$ were the growth rates of Lactobacillus spp. and Bifidobacteria spp. and the hardness of the dairy tofu, respectively. $f_{1}, f_{2}, f_{3}$ represented the modeled response surfaces. $X_{1}, X_{2}, X_{3}, X_{4}$, defined as natural variables, were the concentrations of GDL, peptides, IMO and milk,respectively. $\epsilon_{1}, \epsilon_{2}, \epsilon_{3}$ were the errors in each model. With RSM, it is convenient to transform the natural variables to coded variables $\xi_{1}, \xi_{2}, \xi_{3}, \xi_{4}$, where the coded variables are defined as dimensionless, with mean zero and the same spread or standard deviation:

$Y_{i}=f_{i}\left(\xi_{1}, \xi_{2}, \xi_{3}, \xi_{4}\right)+\epsilon_{i} \quad i=1,2,3$

\section{Optimization on the response surface models and model verifications}

\subsection{Development of the objective function}

All measured variables are subjected to random errors and should be considered in the formulation of the objective function. In order to search a solution maximizing multiple responses, a composite function $(\mathrm{CF})$ was defined as the following:

Composite Function $(\mathrm{CF})=\left(f_{1} \times f_{2} \times f_{3}\right)^{1 / 3}$

The composite function combines three responses into one single function whose maximum can be sought by optimization techniques. Each response contributes equally to the composite function. 


\subsection{Optimization by the sequential quadratic programming}

An SQP procedure implemented in the MATLAB (The Math Works Inc., 2000) environment was employed to optimize the growth rate of probiotics and hardness of probiotic dairy tofu that were formulated, via RSM, as polynomial functions of four independent variables bounded by preset upper and lower limits. The basic scheme of an SQP technique can be expressed in the following steps (Chen, 2003; Reklaintis et al., 1983):

Step 1: Set up and solve a quadratic programming (QP) subproblem, giving a search direction.

Step 2: Test for convergence, stop if it is satisfied.

Step 3: Step forward to a new point along the search direction.

Step 4: Update the Hessian matrix in QP and go to step 1.

In order to search for the global optimum, the concept of multi-start global optimization procedure (Snyman \& Fatti, 1987) was combined with the SQP method. Let $F^{*}$ denote the global maximum and $r$ be the number of sample points falling within the region of convergence of the current overall maximum $F$ after $n$ points have been sampled. Then, under statistically noninformative prior distribution, the probability that $F$ be equal to $F^{*}$ satisfies the following relationship (Chen, 2003):

$$
\begin{aligned}
\operatorname{Pr}[F & \left.=F^{*}\right] \geqq q(n, r) \\
& =1-[(n+1) !(2 n-r) !] /[(2 n+1) !(n-r) !]
\end{aligned}
$$

A very high probability ( $>0.9999)$ in Eq. (5) was set in this study to ensure the global optimum would be attained.

\subsection{Optimization by Genetic Algorithms}

In the present paper, the Simple Genetic Algorithm and the Micro Genetic Algorithm were also employed to optimize the processing conditions. Both GAs were programmed in MATLAB (The Math Works Inc., 2000) codes. The numbers of bits in the binary strings, population size, crossover rate, mutation rate and maximum number of generations in GAs were all obtained by fine tuning the algorithms through multiple trial runs, which is a common practice for using GAs to solve an optimization problem, e.g. D'souza and Simpson (2003) and Vallapuzha et al. (2002).

\subsubsection{Simple Genetic Algorithm}

The Simple Genetic Algorithm (SGA)(Chen et al., 2003; Mitchell, 1996) searches for optimal values by sim- ulating the biological evolutionary process, based on crossover and mutational genetics. In order to use the GAs, a chromosome was formed by all four independent variables, i.e. four ingredients: concentrations of GDL, peptides, IMO and milk, which were all coded as 20bit binary strings making an 80-bit chromosome. Table 2 shows the parameters for the SGA. The initial population, consisting of 50 chromosomes (population size), was generated at random. The crossover and mutation operators were applied to those chromosomes. The crossover rate and mutation rate were 0.5 and 0.02 individually. The selection technique was based on the roulette wheel selection and the elitist strategy (Chen et al., 2003; Mitchell, 1996). The roulette wheel technique is the most simple selection method while the elitist strategy makes sure the one chromosome with the highest $\mathrm{CF}$ value survives to the next generation. The maximum number of generations was set to 100 for SGA.

This iterative process continues until a pre-specified maximum number (100) of generations are reached, or until there is no appreciable improvement in the $\mathrm{CF}$ value. With each new generation, the population gets closer to an optimal value. Once the search is complete, the best value from the final generation is taken as the optimal solution.

\subsubsection{Micro Genetic Algorithm}

The essence of the Micro Genetic Algorithm (MGA) (Chen et al., 2003) is the lack of mutations and the presence of restarts. Due to these features, the algorithm converges rapidly to a local or global maximum (Nikitas, Pappa-Louisi, Papageorgiou, \& Zitrou, 2001). The lack of mutations also results rapidly in a decrease of the variance of the cost values of the population. When the variance value falls below a certain limit, a restarting process begins, in which the chromosome with the highest $\mathrm{CF}$ value is retained and the rest $N-1$ chromosomes ( $N$ is the total number of chromosomes in one generation) are replaced by randomly generated new ones.

Table 2 also shows the parameters for the adopted MGA. The initial population consisting of 10 chromosomes (population size) was generated at random. The crossover rate was 0.5 . The chromosomes with higher $\mathrm{CF}$ values were selected and retained for the next

Table 2

Parameters of the Simple Genetic Algorithm (SGA) and the Micro

\begin{tabular}{|c|c|c|}
\hline Parameter & SGA & MGA \\
\hline Population size & 50 & 10 \\
\hline Number of bits ${ }^{\mathrm{a}}$ & $20 \times 4$ & $20 \times 4$ \\
\hline Mutation rate & 0.02 & 0 \\
\hline Crossover rate & 0.5 & 0.5 \\
\hline Maximum generation & 100 & 500 \\
\hline
\end{tabular}
Genetic Algorithm (MGA)

${ }^{\text {a }}$ No. of bits $=$ No. of bits per variable $\times$ No. of variables. 
generation. The maximum number of generations was set to 500 for the MGA.

\subsection{Model verification}

After optimal processing conditions were found by the SQP and GAs, experiments based on the conditions were performed and repeated three times. The results were then analyzed using ANOVA from the SAS software package (SAS Institute Inc., 1990), with Duncan's multiple range test for significance to detect differences between predicted values and observed values.

\section{Results and discussion}

\subsection{Response surface modeling}

The present work has developed prediction models for the growth rates of probiotics and the hardness of the probiotic dairy tofu by using RSM. Four treatments (concentrations of GDL, peptides, IMO and milk) were mixed with milk in an attempt to improve the growth rate of Lactobacillus spp. and Bifidobacteria spp. as well as the hardness of the product.

The experimental results for the probiotic growth rates and hardness of the probiotic dairy tofu based on a Box-Behnkin design of experiments, and analysis of variance (ANOVA) of their means are presented in Tables 3 and 4. According to the results, approximate functions are constructed using a curve fitting procedure. The model-fitting step was carried out using the Design-Expert ${ }^{\circledR}$ software package, which employs the least squares procedure to compute the model coefficients. The responses modeled as linear, quadratic and cubic functions of the four independent variables were tested for adequacy and for model fitness using ANOVA. The selections of adequate models (Table 4) were determined using model analysis, lack-of fit test and $R$-square analysis as outline by Lee et al. (2000) and Weng et al. (2001). Table 4(a) examines the probability (Prob > F) to see if it falls below 0.05. The highest order polynomial that is significant is selected. The "Lack of Fit Test" (Table 4b) compares the residual error to the

Table 3

Box-Behnkin design matrix with three responses

\begin{tabular}{|c|c|c|c|c|c|c|}
\hline \multicolumn{4}{|c|}{ Independent variables } & \multicolumn{3}{|l|}{ Responses } \\
\hline GDL $\%$ & Peptide $\%$ & $\mathrm{IMO} \%$ & Milk\% & $\overline{\mathrm{GR}^{\mathrm{a}}(\mathrm{L})^{\mathrm{b}}}$ & $\mathrm{GR}(\mathrm{B})^{\mathrm{c}}$ & Hardness $^{\mathrm{d}}$ \\
\hline 0 & 0 & 0 & 0 & $0.153 \pm 0.005$ & $0.124 \pm 0.004$ & $36.18 \pm 3.24$ \\
\hline 0 & -1 & 1 & 0 & $0.145 \pm 0.004$ & $0.163 \pm 0.005$ & $64.42 \pm 5.87$ \\
\hline 0 & 0 & 1 & 1 & $0.174 \pm 0.003$ & $0.125 \pm 0.003$ & $37.60 \pm 2.03$ \\
\hline 1 & 0 & 1 & 0 & $0.173 \pm 0.007$ & $0.123 \pm 0.007$ & $48.86 \pm 4.33$ \\
\hline 0 & 0 & 0 & 0 & $0.165 \pm 0.006$ & $0.124 \pm 0.006$ & $41.18 \pm 4.32$ \\
\hline-1 & 1 & 0 & 0 & $0.192 \pm 0.006$ & $0.123 \pm 0.003$ & $23.12 \pm 5.73$ \\
\hline 1 & 0 & 0 & -1 & $0.131 \pm 0.009$ & $0.116 \pm 0.004$ & $44.90 \pm 3.21$ \\
\hline 0 & 1 & 1 & 0 & $0.162 \pm 0.006$ & $0.133 \pm 0.005$ & $32.45 \pm 2.45$ \\
\hline 0 & -1 & 0 & -1 & $0.134 \pm 0.009$ & $0.124 \pm 0.003$ & $54.17 \pm 5.12$ \\
\hline 1 & 1 & 0 & 0 & $0.152 \pm 0.003$ & $0.135 \pm 0.007$ & $48.86 \pm 1.01$ \\
\hline 0 & 0 & 0 & 0 & $0.175 \pm 0.006$ & $0.123 \pm 0.009$ & $32.32 \pm 3.98$ \\
\hline 0 & 0 & 0 & 0 & $0.166 \pm 0.004$ & $0.107 \pm 0.003$ & $39.99 \pm 2.39$ \\
\hline-1 & 0 & 1 & 0 & $0.174 \pm 0.003$ & $0.124 \pm 0.002$ & $28.89 \pm 3.25$ \\
\hline 1 & 0 & 0 & 1 & $0.153 \pm 0.005$ & $0.116 \pm 0.003$ & $54.30 \pm 3.45$ \\
\hline 0 & -1 & 0 & 1 & $0.133 \pm 0.007$ & $0.134 \pm 0.002$ & $82.70 \pm 2.11$ \\
\hline-1 & 0 & 0 & -1 & $0.183 \pm 0.005$ & $0.115 \pm 0.003$ & $29.24 \pm 3.53$ \\
\hline 0 & 1 & 0 & 1 & $0.156 \pm 0.007$ & $0.117 \pm 0.001$ & $35.86 \pm 4.21$ \\
\hline-1 & 0 & 0 & 1 & $0.195 \pm 0.007$ & $0.123 \pm 0.003$ & $37.15 \pm 5.23$ \\
\hline-1 & 0 & -1 & 0 & $0.183 \pm 0.006$ & $0.138 \pm 0.003$ & $36.64 \pm 3.27$ \\
\hline 0 & 0 & 0 & 0 & $0.163 \pm 0.008$ & $0.104 \pm 0.002$ & $39.59 \pm 2.31$ \\
\hline 0 & 0 & -1 & -1 & $0.175 \pm 0.005$ & $0.135 \pm 0.001$ & $42.59 \pm 4.54$ \\
\hline 0 & 1 & 0 & -1 & $0.153 \pm 0.005$ & $0.103 \pm 0.002$ & $33.81 \pm 2.32$ \\
\hline 0 & 1 & -1 & 0 & $0.187 \pm 0.006$ & $0.165 \pm 0.001$ & $43.36 \pm 3.17$ \\
\hline-1 & -1 & 0 & 0 & $0.156 \pm 0.007$ & $0.134 \pm 0.002$ & $49.30 \pm 3.02$ \\
\hline 1 & 0 & -1 & 0 & $0.178 \pm 0.003$ & $0.124 \pm 0.002$ & $58.15 \pm 4.35$ \\
\hline 0 & 0 & 1 & -1 & $0.165 \pm 0.004$ & $0.123 \pm 0.003$ & $56.35 \pm 1.43$ \\
\hline 0 & -1 & -1 & 0 & $0.143 \pm 0.007$ & $0.146 \pm 0.002$ & $61.74 \pm 2.23$ \\
\hline 1 & -1 & 0 & 0 & $0.145 \pm 0.005$ & $0.154 \pm 0.004$ & $77.90 \pm 3.48$ \\
\hline 0 & 0 & -1 & 1 & $0.184 \pm 0.006$ & $0.103 \pm 0.005$ & $39.25 \pm 1.32$ \\
\hline
\end{tabular}

${ }^{\text {a }}$ GR: growth rate.

b L: L. acidophilus + L. casei.

c B: B. longum + B. bifidum.

d Hardness: Unit in g. 
Table 4

(a) Model analysis, (b) lack of fit and (c) $R$-square analysis of probiotic growth rate model after 12-h fermentation

\begin{tabular}{|c|c|c|c|c|c|c|}
\hline \multirow[t]{2}{*}{ Source } & \multicolumn{2}{|l|}{$\mathrm{AC}^{\mathrm{a}}$ growth rate } & \multicolumn{2}{|l|}{$\mathrm{B}^{\mathrm{b}}$ growth rate } & \multicolumn{2}{|l|}{ Hardness } \\
\hline & Sum of squares & $P>F$ & Sum of squares & $P>F$ & Sum of squares & $P>F$ \\
\hline \multicolumn{7}{|c|}{ (a) Model analysis ${ }^{\mathrm{c}}$} \\
\hline Mean & 0.74 & $0.0030^{* *}$ & 0.43 & $0.0021^{* *}$ & 59256.57 & $<0.0001^{* *}$ \\
\hline Linear & $4.930 \times 10^{-3}$ & $0.0002^{* *}$ & $5.967 \times 10^{-4}$ & 0.6469 & 3936.02 & $<0.0001^{* *}$ \\
\hline Quadratic & $2.506 \times 10^{-3}$ & $0.0212^{*}$ & $3.996 \times 10^{-3}$ & $0.0211^{*}$ & 989.09 & 0.0924 \\
\hline Cubic & $7.527 \times 10^{-4}$ & 0.2476 & $8.156 \times 10^{-4}$ & 0.6943 & 583.00 & $0.0135^{*}$ \\
\hline Residual & $3.159 \times 10^{-4}$ & & $8.862 \times 10^{-4}$ & & 60.67 & \\
\hline Total & 0.75 & & 0.44 & & 64825.35 & \\
\hline \multicolumn{7}{|c|}{ (b) Lack of fit ${ }^{\mathrm{d}}$} \\
\hline Linear & $3.447 \times 10^{-3}$ & 0.0561 & $5.280 \times 10^{-3}$ & 0.1906 & 1580.61 & $0.0463^{*}$ \\
\hline Quadratic & $9.416 \times 10^{-4}$ & 0.1531 & $1.284 \times 10^{-3}$ & 0.4553 & 591.52 & 0.0790 \\
\hline Cubic & $1.889 \times 10^{-4}$ & 0.1615 & $4.684 \times 10^{-4}$ & 0.2222 & 8.52 & 0.7387 \\
\hline Pure error & $1.270 \times 10^{-4}$ & & $4.178 \times 10^{-4}$ & & 52.14 & \\
\hline \multicolumn{7}{|c|}{ (c) R-square analysis $\mathrm{e}^{\mathrm{e}}$} \\
\hline & $R$-square & Press & $R$-square & Press & $R$-squared & Press \\
\hline Linear & 0.5797 & $5.538 \times 10^{-3}$ & 0.0948 & $8.562 \times 10^{-3}$ & 0.7068 & 2399.16 \\
\hline Quadratic & 0.8743 & $5.622 \times 10^{-3}$ & 0.7296 & $8.049 \times 10^{-3}$ & 0.8844 & 3488.66 \\
\hline Cubic & 0.9629 & 0.027 & 0.8592 & 0.068 & 0.9891 & 1309.02 \\
\hline
\end{tabular}

*Significant at 5\% level.

** Significant at $1 \%$ level.

${ }^{a}$ AC: L. acidophilus + L. casei.

b $\mathrm{B}:$ B. longum + B. bifidum.

c Model analysis: select the highest order polynomial where the additional terms are significant.

d Lack of fit test: want the selected model to have insignificant lack-of-fit.

e $R$-square analysis: focus on the model minimizing the "Press".

pure error from replicated design points. If there is a significant lack of fit, as indicated by a low probability value (Prob > F), the response predictor should be discarded. The model with insignificant lack-of-fit is selected. ANOVA showed that the quadratic models of Eq. (6) for the growth rates of Lactobacillus spp. and Bifidobacteria spp. as well as the cubic model of Eq. (7) for the hardness of the product appeared to be the most accurate, with no significant lack of fit (Table 4).

$$
\begin{aligned}
f_{k}= & \beta_{0}+\sum_{i=1}^{n} \beta_{i} X_{i}+\sum_{i=1}^{n} \beta_{i i} X_{i}^{2}+\sum_{i=1}^{n-1} \sum_{j=i+1}^{n} \beta_{i j} X_{i} X_{j} \\
k & =1,2 \\
f_{3}= & \beta_{0}+\sum_{i=1}^{n} \beta_{i} X_{i}+\sum_{i=1}^{n} \beta_{i i} X_{i}^{2}+\sum_{i=1}^{n-1} \sum_{j=i+1}^{n} \beta_{i j} X_{i} X_{j} \\
& +\sum_{i=1}^{n} \beta_{i i i} X_{i}^{3}+\sum_{i=1}^{n-1} \sum_{j=i+1}^{n} \beta_{i i j} X_{i}^{2} X_{j}+\sum_{i=1}^{n-1} \sum_{j=i+1}^{n} \beta_{i j j} X_{i} X_{j}^{2} \\
& +\sum_{i=1}^{n-2} \sum_{j=i+1}^{n-1} \sum_{k=j+1}^{n} \beta_{i j k} X_{i} X_{j} X_{k}
\end{aligned}
$$

where $f_{1}$ and $f_{2}$ were the growth rates of Lactobacillus spp. and Bifidobacteria spp., respectively, and $f_{3}$ was the hardness of the product. $\beta_{0}, \beta_{i}, \beta_{i i}, \beta_{i j}, \beta_{i i i}, \beta_{i i j}, \beta_{i j j}$, $\beta_{i j k}$ were constant coefficients and $X_{i}, X_{j}, X_{k}$ were the uncoded independent variables. The regression coeffi- cients for the statistically significant models are given in Table 5. The three-level design of BBD is incapable of forming the pure cubic terms, i.e. $\beta_{i i i} X_{i}^{3}$ in Eq. (7), and the coefficients in Table 5 do concur with this fact. The three responses were then combined into one single function (Composite Function) whose maximum can then be sought by optimization techniques.

\subsection{Search for optimal combinations of ingredients}

\subsubsection{Sequential quadratic programming}

Since the composite function was a product of two quadratic and one cubic functions, it was very likely that there existed more than one local maximum. Therefore, a global optimization program consisting of a multistart SQP with the probability criterion for global optimum, i.e. Eq. (5), was coded. The program generated a series of uniformly distributed random points as initial searching points, and then SQP was applied to find the optimum based on each initial points. If the probability exceeds a preset value $(99.99 \%$, in this study) according to Eq. (5), the global optimum is considered found. Otherwise, next random initial point is generated and SQP executed again. Table 6 shows the initial points and their corresponding optimal CF values and Fig. 1 shows the evolution of all optimal values graphically. From Table 6 , there are six different local optimal CF values (from 1.0131 to 1.2944 ) found from 28 randomly generated 
Table 5

The coefficients of probiotic growth rate model and hardness model of probiotic dairy tofu after 12 -h fermentation

\begin{tabular}{|c|c|c|c|}
\hline Coefficient $\backslash Y$ & $\mathrm{GR}^{\mathrm{a}}(\mathrm{AC})^{\mathrm{b}}$ & $\operatorname{GR}(B)^{c}$ & Hardness \\
\hline$\beta_{0}$ & 0.10 & -0.066 & -134.81 \\
\hline$B_{1}$ & -0.098 & $-4.557 \times 10^{-3}$ & 503.52 \\
\hline$B_{2}$ & 0.046 & -0.048 & 208.61 \\
\hline$\beta_{3}$ & -0.013 & -0.033 & 4.26 \\
\hline$B_{4}$ & $9.645 \times 10^{-3}$ & 0.030 & 7.68 \\
\hline$\beta_{11}$ & 0.037 & 0.016 & -354.42 \\
\hline$\beta_{22}$ & -0.021 & 0.027 & -107.43 \\
\hline$B_{33}$ & $4.007 \times 10^{-3}$ & $5.176 \times 10^{-3}$ & -2.27 \\
\hline$\beta_{44}$ & $-3.306 \times 10^{-4}$ & $-1.053 \times 10^{-3}$ & 0.31 \\
\hline$\beta_{12}$ & -0.030 & $-2.393 \times 10^{-3}$ & -86.11 \\
\hline$\beta_{13}$ & $5.871 \times 10^{-3}$ & $7.610 \times 10^{-3}$ & 42.58 \\
\hline$\beta_{14}$ & $2.021 \times 10^{-3}$ & $-1.537 \times 10^{-3}$ & -34.46 \\
\hline$\beta_{23}$ & $-4.855 \times 10^{-3}$ & $-9.272 \times 10^{-3}$ & -1.47 \\
\hline$\beta_{24}$ & $2.048 \times 10^{-3}$ & $8.749 \times 10^{-4}$ & -14.65 \\
\hline$B_{34}$ & $-1.902 \times 10^{-4}$ & $1.305 \times 10^{-3}$ & -0.86 \\
\hline$\beta_{111}$ & 0 & 0 & 0 \\
\hline$B_{222}$ & 0 & 0 & 0 \\
\hline$B_{333}$ & 0 & 0 & 0 \\
\hline$B_{444}$ & 0 & 0 & 0 \\
\hline$B_{112}$ & 0 & 0 & 32.61 \\
\hline$\beta_{113}$ & 0 & 0 & -39.66 \\
\hline$B_{114}$ & 0 & 0 & 26.78 \\
\hline$B_{122}$ & 0 & 0 & 27.32 \\
\hline$\beta_{133}$ & 0 & 0 & 2.75 \\
\hline$B_{144}$ & 0 & 0 & 0 \\
\hline$B_{223}$ & 0 & 0 & -6.03 \\
\hline$B_{224}$ & 0 & 0 & 7.80 \\
\hline$B_{233}$ & 0 & 0 & 2.50 \\
\hline$B_{244}$ & 0 & 0 & 0 \\
\hline$B_{334}$ & 0 & 0 & 0 \\
\hline$B_{344}$ & 0 & 0 & 0 \\
\hline$B_{123}$ & 0 & 0 & 0 \\
\hline$\beta_{124}$ & 0 & 0 & 0 \\
\hline$\beta_{134}$ & 0 & 0 & 0 \\
\hline$B_{234}$ & 0 & 0 & 0 \\
\hline
\end{tabular}

${ }^{a} \mathrm{GR}$ : growth rate.

${ }^{b}$ AC: $L$. acidophilus + L. casei.

${ }^{\text {c }}$ B: B. longum + B. bifidum.

initial points. The rightmost column of Table 6 shows the probability of an optimal CF value being the global one. Among those local optimal values, the global optimal CF value was 1.2944 with $99.99 \%$ certainty. The optimal CF value corresponds to 0.1463 of growth rate for Lactobacillus spp., 0.1575 of growth rate for Bifidobacteria spp. and 94.15 of the product's hardness. There were 11 sets among 28 sets attained the highest optimal CF value (1.2944) with optimal points $X_{1}=1, X_{2}=0$, $X_{3}=3$ and $X_{4}=18$. The optimal conditions for manufacturing probiotic dairy tofu were $18 \%$ of skim milk blended with $1 \%$ of GDL, $0 \%$ of peptides and $3 \%$ of IMO.

To further depict the global optimization results, 3-D response surface plots were created by fixing two of the four factors. Fig. 2 shows four local maxima including the global one in a CF response function that was produced by setting $X_{1}=1$ and $X_{4}=18$ while varying $X_{2}$ and $X_{3}$ within their boundaries. Fig. 3 shows the other two local maxima found during the optimization process by fixing $X_{3}=0$ and $X_{4}=18$ while changing $X_{1}$ and $X_{2}$. The optimization results clearly show that whether the global optimum could be found depends on the initial searching points for our response surface models. To reassure the global optimum that was found was indeed the global one, GAs were also applied on the CF response surface model.

\subsubsection{Genetic Algorithms}

The composite function was optimized using the SGA and MGA. Fig. 4 shows the evolution curves in searching for the global optimum. The composite function value increased in accordance with the number of function evaluations and reached the maximum value on the both curves. The maximum $\mathrm{CF}$ value provided the optimal processing conditions for the probiotic dairy tofu. The number of function evaluations in Eq. (8) represents the efficiency of the algorithms. A smaller number indicates a greater efficiency.

$N_{\mathrm{e}}=N_{\mathrm{g}} \times N_{\mathrm{p}}$

where $N_{\mathrm{e}}, N_{\mathrm{g}}$ and $N_{\mathrm{p}}$ represent, respectively, the numbers of total function evaluations, the generations and the population size.

Both searching procedures were allowed to continue to reach the maximum number of function evaluations, i.e. 5000, even though there had been no significant changes in CF values after 3750 function evaluations. In Fig. 4, both SGA and MGA produced fast increasing $\mathrm{CF}$ values during the early stage of the optimization processes, which are typical for GAs. For 900 function evaluations, the composite function by SGA has been increased from 1.1446 to 1.2834 , compared to 1.0298 to 1.2928 for MGA. The same maximal value (CF value $=1.2944)$ was obtained in 4600 and 3340 function evaluations for SGA and MGA, respectively. The MGA converged more rapidly to the optimal value than did the SGA. The essences of MGA are the lack of mutations and the mechanism of restarts. Due to these features, the algorithm converges faster to the global maximum.

Population size also affected the results (Fig. 4). An optimal value was obtained at the 92nd generation, i.e. $4600 / 50=92$ from Eq. (6), for the SGA curve. For the MGA curve, comparing to the SGA curve, an optimal value was obtained at the 334th generation, i.e. 3340/ $10=334$. This means the highest fitness value could be obtained at earlier generations for increasing population size because of the variety of chromosomes. Moriyama and Shimizu (1996) also drew a similar conclusion and indicated that large population size could decrease the generation to reach the highest fitness value.

The elitist strategy used in this study has been known as an effective way for improving the fitness of 
Table 6

The randomly generated, initial searching points and optimal CF values found by SQP

\begin{tabular}{|c|c|c|c|c|c|c|c|c|c|c|}
\hline \multirow[t]{2}{*}{ Set no. } & \multicolumn{4}{|c|}{ Initial searching point } & \multicolumn{4}{|c|}{ Optimal point } & \multirow[t]{2}{*}{ Optimal CF value } & \multirow[t]{2}{*}{ Probability } \\
\hline & $\overline{\mathrm{X}_{1}^{\mathrm{a}}}$ & $\mathrm{X}_{2}^{\mathrm{b}}$ & $\mathrm{X}_{3}{ }^{\mathrm{c}}$ & $\mathrm{X}_{4}^{\mathrm{d}}$ & $X_{1}$ & $X_{2}$ & $X_{3}$ & $X_{4}$ & & \\
\hline 1 & 0.6911 & 1.0511 & 1.0385 & 12.0752 & 1 & 0 & 0 & 18 & 1.2381 & 0.6667 \\
\hline 2 & 0.8479 & 1.2047 & 0.5282 & 13.8055 & 1 & 1.5 & 0 & 18 & 1.2403 & 0.7000 \\
\hline 3 & 0.3985 & 0.7750 & 0.2037 & 17.7905 & 0.3 & 0 & 0 & 18 & 1.1485 & 0.7143 \\
\hline 4 & 0.6904 & 0.7540 & 0.9282 & 12.6125 & 1 & 0 & 0 & 18 & 1.2381 & 0.7222 \\
\hline 5 & 0.4438 & 1.1321 & 1.0043 & 15.5761 & 0.3 & 1.5 & 0 & 18 & 1.1779 & 0.7273 \\
\hline 6 & 0.9023 & 0.3572 & 1.1285 & 14.8139 & 1 & 0 & 0 & 18 & 1.2381 & 0.7308 \\
\hline 7 & 0.4801 & 1.4146 & 2.8566 & 16.304 & 1 & 1.5 & 3 & 18 & 1.0131 & 0.7333 \\
\hline 8 & 0.4943 & 0.1329 & 2.158 & 17.1532 & 1 & 0 & 3 & 18 & 1.2944 & 0.7353 \\
\hline 9 & 0.5017 & 0.8145 & 2.338 & 13.1136 & 1 & 0 & 3 & 18 & 1.2944 & 0.8762 \\
\hline 10 & 0.8143 & 1.3552 & 1.853 & 14.7742 & 1 & 1.5 & 0 & 18 & 1.2403 & 0.8759 \\
\hline 11 & 0.6479 & 1.2920 & 1.9477 & 17.4198 & 1 & 1.5 & 0 & 18 & 1.2403 & 0.8758 \\
\hline 12 & 0.3383 & 0.9472 & 2.2688 & 12.1326 & 1 & 0 & 3 & 18 & 1.2944 & 0.9435 \\
\hline 13 & 0.4339 & 0.3935 & 0.4435 & 16.5624 & 0.3 & 0 & 0 & 18 & 1.1485 & 0.9430 \\
\hline 14 & 0.8072 & 0.5685 & 1.7984 & 16.7751 & 1 & 0 & 3 & 18 & 1.2944 & 0.9747 \\
\hline 15 & 0.9816 & 0.5060 & 2.6958 & 13.8831 & 1 & 0 & 3 & 18 & 1.2944 & 0.9891 \\
\hline 16 & 0.7931 & 0.4719 & 0.5158 & 13.415 & 1 & 0 & 0 & 18 & 1.2381 & 0.9888 \\
\hline 17 & 0.8611 & 1.4207 & 2.4568 & 14.9667 & 1 & 1.5 & 3 & 18 & 1.0131 & 0.9886 \\
\hline 18 & 0.4444 & 0.8028 & 0.2079 & 16.1115 & 0.3 & 0 & 0 & 18 & 1.1485 & 0.9883 \\
\hline 19 & 0.8409 & 1.0529 & 2.8671 & 17.6678 & 1 & 0 & 3 & 18 & 1.2944 & 0.9950 \\
\hline 20 & 0.9109 & 1.3126 & 0.952 & 14.6359 & 1 & 1.5 & 0 & 18 & 1.2403 & 0.9948 \\
\hline 21 & 0.8652 & 1.4794 & 0.0156 & 14.9846 & 1 & 1.5 & 0 & 18 & 1.2403 & 0.9947 \\
\hline 22 & 0.8296 & 1.3280 & 2.2798 & 12.5917 & 1 & 0 & 3 & 18 & 1.2944 & 0.9977 \\
\hline 23 & 0.9332 & 0.6073 & 0.926 & 15.3266 & 1 & 0 & 0 & 18 & 1.2381 & 0.9977 \\
\hline 24 & 0.5947 & 0.9407 & 2.1458 & 14.6142 & 1 & 0 & 3 & 18 & 1.2944 & 0.9990 \\
\hline 24 & 0.7609 & 0.5782 & 0.2428 & 13.0876 & 1 & 0 & 0 & 18 & 1.2381 & 0.9990 \\
\hline 26 & 0.3009 & 1.2718 & 2.5376 & 12.9229 & 1 & 0 & 3 & 18 & 1.2944 & 0.9996 \\
\hline 27 & 0.9874 & 0.7903 & 2.1551 & 17.6024 & 1 & 0 & 3 & 18 & 1.2944 & 0.9998 \\
\hline 28 & 0.5886 & 1.2111 & 2.6112 & 12.8176 & 1 & 0 & 3 & 18 & 1.2944 & 0.9999 \\
\hline
\end{tabular}

chromosomes because a chromosome with maximum fitness is compulsorily remained for next generation (Morimoto, Purwanto, Suzuki, \& Hashimoto, 1997).

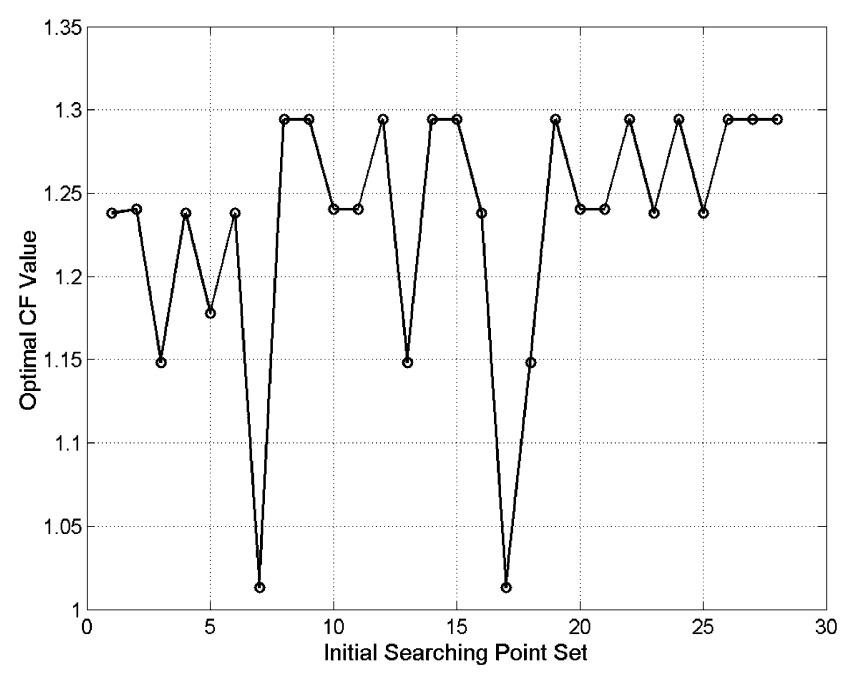

Fig. 1. Optimum $\mathrm{CF}$ values for randomly generated initial searching points when using SQP.

\subsubsection{Comparison between $G A s$ and $S Q P$}

Comparing the optimization results, the SQP and GAs all produced the global optimum in this research. Both GAs yielded comparable results with MGA being

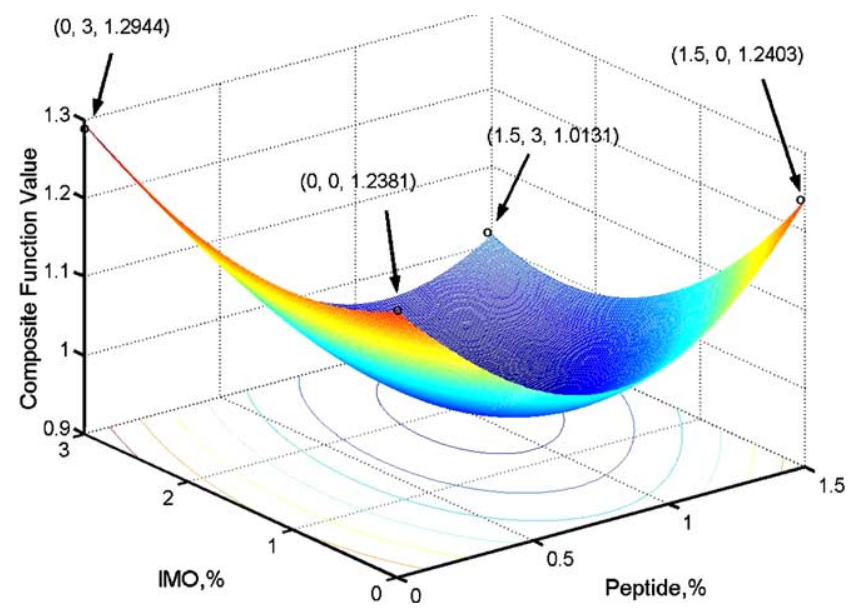

Fig. 2. A response surface plot under the conditions of constant GDL $(1 \%)$ and skim milk $(18 \%)$. 


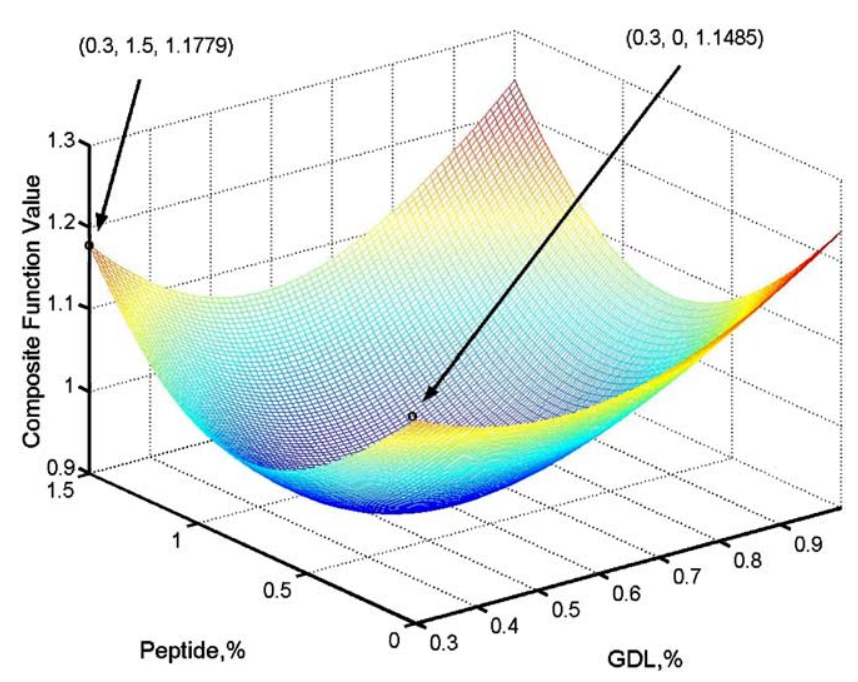

Fig. 3. A response surface plot under the conditions of constant IMO $(0 \%)$ and skim milk $(18 \%)$.

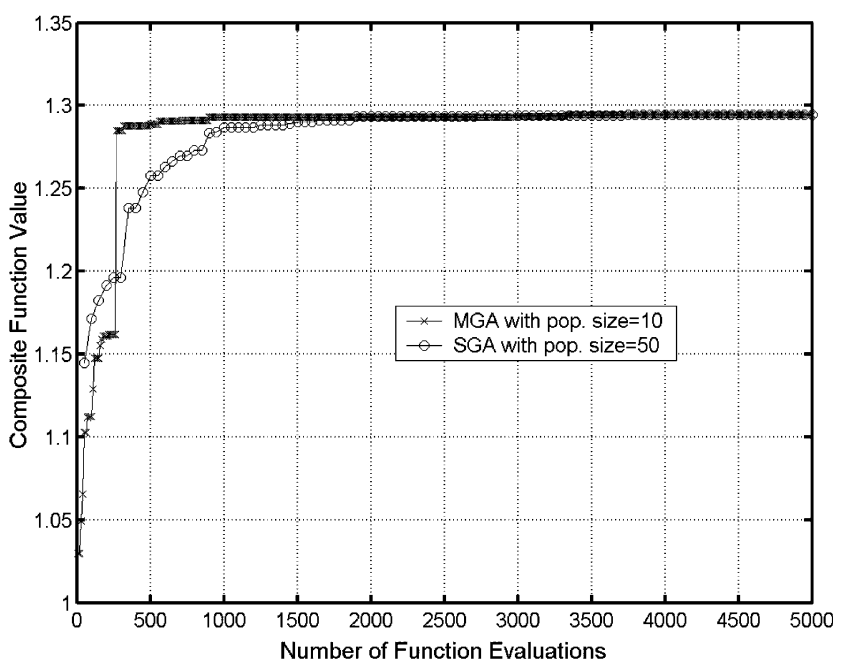

Fig. 4. Evolution curves of the two Genetic Algorithms for searching the optimal processing conditions for the probiotic dairy tofu.

slightly more efficient than SGA. Although the SQP's searching performance is dictated by the initial searching points in our study, the modified SQP with the multi-start capability reached the global optimum with $99.99 \%$ certainty in 28 tries. The SQP was obviously the most efficient method of all.

\subsubsection{Effects of optimized factors on responses}

The optimal manufacturing conditions were found to be $1 \%$ of GDL (upper limit), 3\% of IMO (upper limit), $0 \%$ of peptides (lower limit) and $18 \%$ of milk concentration (upper limit). Addition of skim milk powder can increase the total solid of milk and improve the hardness of dairy tofu. GDL is allowed for use in human food as a coagulant and a $\mathrm{pH}$ control agent. Increasing the GDL level can raise the hardness of probiotic dairy tofu.
Therefore, both milk concentration and GDL in their upper limits were suggested by the optimization methods. As for IMO and peptides, both are growth promoters for probiotics. IMO can stimulate lactic microflora as well as facilitate the elevated product of butyrate (Fooks, Fuller, \& Gibson, 1999). Consequently, the highest level of IMO was recommended by the optimization procedures. Peptides have been proved to be able to improve the viability of bifidobacteria (Dave \& Shah, 1997). According to our preliminary tests, peptides did increase the growth rate of bifidobacteria, but decreased the hardness of dairy tofu. The composite function (Eq. (4)) is composed of all three responses. If the addition of a certain factor increases one response but decreases another, a compromise will be made to achieve a higher composite function value during optimization. This is why peptides attained the value of the lower limit in our results. The optimal conditions are the results of the interactions among the four factors and the combined effects of all three responses.

\subsection{Experimental verification}

The optimal producing conditions were suggested by the MGA, SGA and SQP, and were verified by additional independent experiments. The optimal manufacturing conditions were obtained as those of maximum GDL $(1 \%)$, IMO (3\%) as well as milk concentrations (18\%), and in combination with a minimal peptides level $(0 \%)$. The three responses, the growth rates of Lactobacillus spp. and Bifidobacteria spp. and the hardness of the dairy tofu, and the composite function value produced by verification experiments were all very close to the predicted values with no apparent significant differences being demonstrated between the two sets $(P>0.05)$.

\section{Conclusion}

The two-stage effort of obtaining a surface model using RSM, and optimizing this model using GAs or SQP techniques has been demonstrated to represent an effective approach. Both SQP and GAs techniques are able to determine the optimal conditions for manufacturing the probiotic dairy tofu. The conditions were $1 \%$ of GDL, $0 \%$ of peptides level, $3 \%$ of IMO and $18 \%$ of milk concentrations, and they were confirmed by verification experiments. Among the SQP and two GAs employed, the SQP, modified with the multi-start capability, is the most efficient one.

\section{Acknowledgment}

The authors wish to thank the National Science Council of Taiwan, ROC (NSC90-2313-B254-001) for their support of this research. 


\section{References}

Arora, J. S. (1989). Introduction to optimum design. New York: McGraw-Hill.

Box, G. E. P., \& Behnkin, E. W. (1960). Some new three level designs for the study of quantitative variables. Technometrics, 2, 455-475.

Chen, M. J., \& Lin, C. W. (2002). Factors affecting the water-holding capacity of fibrinogen/plasma protein gels optimized by response surface methodology. Journal of Food Science, 67(7), 2579-2582.

Chen, M. J., Chen, K. N., \& Lin, C. W. (2003). Optimization of the viability of probiotics in a new fermented milk drink by Genetic Algorithms for response surface modeling. Journal of Food Science, 68(2), 632-638.

Chen, S. (2003). Robust design with dynamic characteristics using stochastic Sequential Quadratic Programming. Engineering Optimization, 35(1), 79-89.

Dave, R. I., \& Shah, N. P. (1997). Ingredient supplementation effects on viability of probiotic bacteria in yogurt. Journal of Dairy Science, 81, 2804-2816.

D'souza, B., \& Simpson, T. W. (2003). A Genetic Algorithm based method for product family design optimization. Engineering Optimization, 35(1), 1-18.

Fooks, L. J., Fuller, R., \& Gibson, G. R. (1999). Prebiotics, probiotics and human gut microbiology. International Dairy Journal, 9, 53-61.

Haftka, R. T., \& Gürdal, Z. (1992). Elements of structural optimization (Third revised and expanded edition). The Netherlands: Kluwer Academic Publishers.

Lapierre, L., Undeland, P., \& Cox, L. J. (1992). Lithium chloridesodium propionate agar for the enumeration of bifidobacteria in fermented dairy products. Journal of Dairy Science, 75, 1192-1196.

Lee, J., Ye, L., Landen, W. O., \& Eitenmiller, R. R. (2000). Optimization of an extraction procedure for the quantification of vitamin $\mathrm{E}$ in tomato and broccoli using response surface methodology. Journal of Food Composition and Analysis, 13, 45-57.

Liu, Y., Chen, M., \& Lin, W. (2002). Studies on Lao-Chao culture filtrate for a flavoring agent in a yogurt-like product. AsianAustralasian Journal of Animal Sciences, 15(3), 172-179.

Mitchell, M. (1996). An introduction to Genetic Algorithms. London, England: The MIT Press.
Morimoto, T., Purwanto, W., Suzuki, J., \& Hashimoto, Y. (1997). Optimization of heat treatment for fruit during storage using neural networks and Genetic Algorithms. Computers and Electronics in Agriculture, 19, 89-101.

Moriyama, J., \& Shimizu, K. (1996). Online optimization of culture temperature for ethanol fermentation using a Genetic Algorithm. Journal of Chem Tech Biotechnol, 66, 217-222.

Myers, R. H., \& Montgomery, D. C. (1995). Response surface methodology: process and product optimization using designed experiments (1st ed.). New York: John Wiley \& Sons, Inc.

Nikitas, P., Pappa-Louisi, A., Papageorgiou, A., \& Zitrou, A. (2001). On the use of Genetic Algorithms for response surface modeling in highperformance liquid chromatography and their combination with the Microsoft Solver. Journal of Chromatography A, 942, 93-105.

Porretta, A., Birzi, A. C., \& Vicini, E. (1995). Effects of ultra-high hydrostatic pressure treatments on the quality of tomato juice. Food Chemistry, 52, 35-41.

Reklaintis, G. V., Ravindran, A., \& Ragsdell, K. M. (1983). Engineering optimization: methods and applications. New York, USA: John Wiley and Sons.

SAS Institute Inc. (1990). SAS/STAT User's Guide, SAS Institute Inc., North Carolina, USA.

Snyman, J. A., \& Fatti, L. P. (1987). A multi-start global minimization algorithm with dynamic search trajectories. Journal of Optimization Theory and Applications, 54(1), 121-141.

Stat-Ease, Inc. (2000). Design Expert User's Guide, The State-Ease. Inc., USA.

The Math Works Inc. (2000). Using MATLAB. The Math Works Inc., MA, USA.

Vallapuzha, S. E., Meter, C. D., Choudhuri, S., \& Khetan, R. P. (2002). An investigation into the use of spatial coordinates for the Genetic Algorithm based solution of the fixture layout optimization problem. International Journal of Machine Tools and Manufacture, 42, 265-275.

Wang, Q. J. (1997). Using Genetic Algorithms to optimize model parameters. Environ Model Software, 12, 27-34.

Weng, W., Liu, W., \& Lin, W. (2001). Studies on the optimum models of the dairy product Kou Woan Lao using response surface methodology. Asian-Australasian Journal of Animal Sciences, 14(10), 1470-1476. 